\title{
Solution of Singular Integrals in Mathematical Model of Mode I Crack Near Strength Mismatched Interface
}

\author{
Sunil Bhat ${ }^{1}$, Vijay G. Ukadgaonker ${ }^{2}$ \\ ${ }^{1}$ School of Mechanical and Building Sciences, VIT University, Vellore, India \\ ${ }^{2}$ Department of Mechanical Engineering, Indian Institute of Technology, Mumbai, India \\ Email: sbhat_789@rediffmail.com
}

Received April 5, 2012; revised May 2, 2012; accepted May 10, 2012

\begin{abstract}
Characteristics of Mode I crack near the interface of elasticity matched but plasticity and strength mismatched materials differ from those of the crack in a homogenous body. Interface body of different strength influences the plastic or cohesive zone at the crack tip in parent body. The mathematical model for load line opening of the crack near the interface in linear elastic regime involves singular integrals. The paper presents explicit solution of these integrals with the help of Cauchy's principal value theorem. Cases of thin and thick welds between the materials are investigated. Solutions of the integrals are well substantiated. Final results are provided in a consolidated form.
\end{abstract}

Keywords: Crack Opening Displacement; Singular Integrals; Strength Mismatch; Weld Interface; Cauchy's Principal Value Theorem

\section{Introduction}

The material behaviour at the tip of the Mode I crack in a homogenous body is in general very complex and difficult to describe by continuum mechanical models. The crack tip region where the material undergoes degradation or damage is known as the process region. Refer Figure 1(a). Micro-mechanical processes, viz. microcracking in brittle materials and void initiation and coalescence in ductile materials create new traction free surfaces or cracks in process region. Yielding occurs outside the process region. This zone is called as the plastic or cohesive zone. Cohesive zone is considered as the crack extension under the action of closing cohesive stress generated by elastic constraint exerted by surrounding non-yielded material over the cohesive zone. The cohesive stress is assumed equal to material yield strength in plane stress and $\sqrt{3}$ times the yield strength in plane strain conditions. Qualitative characteristics of the cohesive zone were experimentally verified by Hahn et al. [1]. They conducted experiments on cracked steel specimens and found the cohesive zone, as shown in Figure 1(b), by etching the polished surface in front of the crack tip.

In a bimaterial comprising elasticity identical but plasticity and strength mismatched constituents (like steels), the Mode I crack near the interface has the characteristics similar to the one in homogeneous parent body as long as the cohesive zone is in the parent body alone. The effect of approaching interface body of different strength is not felt by the crack in such a stage because of similar elastic properties across the interface. But as the crack grows and reaches nearer to the interface, the increasing magnitude of crack tip stress field causes the cohesive zone to develop in the interface body. Consequently, the part of cohesive zone in the interface body is subjected to cohesive stress different from that acting over its portion in the parent body that triggers the effect of strength mismatch across the interface over the crack tip. The effect continues with increasing intensity as the cohesive zone spreads deeper into the interface body with crack growth and reaches the maximum when the crack tip touches the interface body with the cohesive zone fully in the interface body.

Cases of thin and thick welds between the steels are examined. Thin weld, obtained by non-fusion, solid state like friction welding between dissimilar steels leads to a single thin interface whereas a thick weld by fusion bonding from electron or laser beam welding results in two interfaces, one between the parent body and the weld and the other between the weld and the interface body. The parent body, the weld and the interface body have similar elastic properties but variable strengths of comparable magnitudes.

\section{Problem Definition}

Solution for load line opening of the crack is obtained by modeling its cohesive zone. Complex potentials are used 


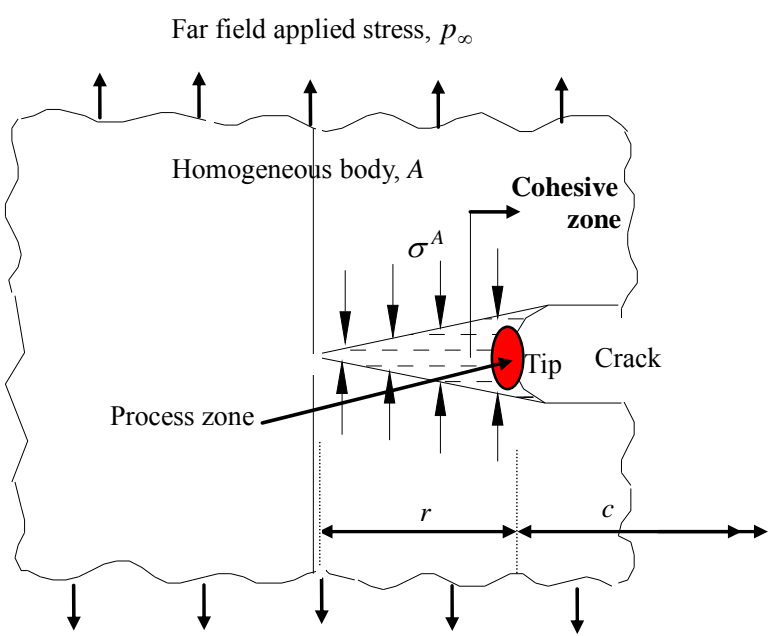

(a)

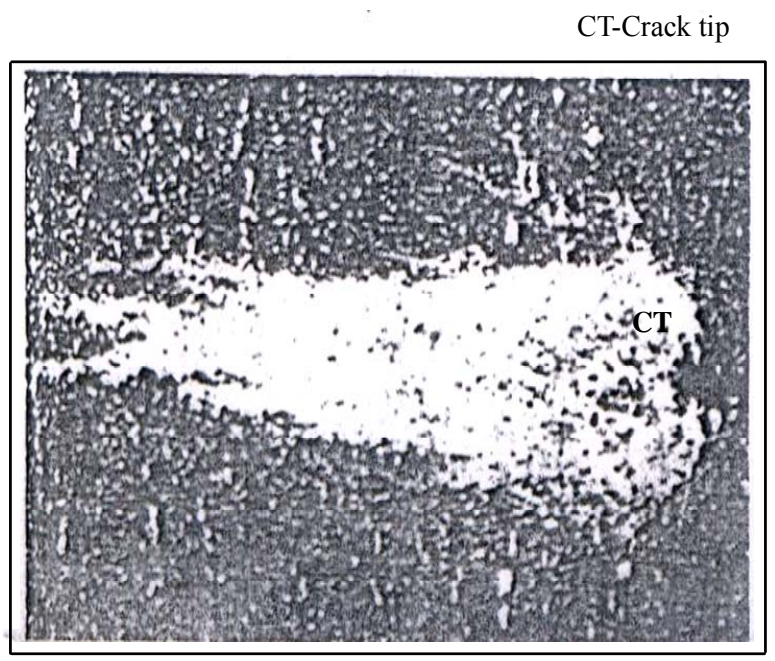

(b)

Figure 1. Crack tip cohesive zone and its experimental validation; (a) Crack tip cohesive zone; (b) Cohesive zone observed experimentally by Hahn et al. [1].

for this purpose in linear elastic regime.

\subsection{Crack Near Thin Weld (Refer Figure 2)}

Half load line crack opening, $v$, in the cohesive zone of size, $r$, in parent body $A$ in stage I, Figure 2(a), subjected to cohesive stress, $\sigma^{A}$, under far field applied stress intensity parameter, $K_{\text {applied }}$, is of the following form [2]:

$$
\frac{\partial v}{\partial x}=-\frac{2}{E}\left\{\frac{K_{\text {applied }}}{\sqrt{2 \pi(r-x)}}+\frac{1}{\pi \sqrt{r-x}} \int_{0}^{r} \frac{\sigma^{A} \sqrt{r-\xi}}{\xi-x} \mathrm{~d} \xi\right\}
$$

where, $E^{A}=E$, is the modulus of elasticity of parent body, $A$. On integrating Equation (1), the expression for $v$, as the function of distance $x$ from the crack tip in the cohesive zone is obtained as

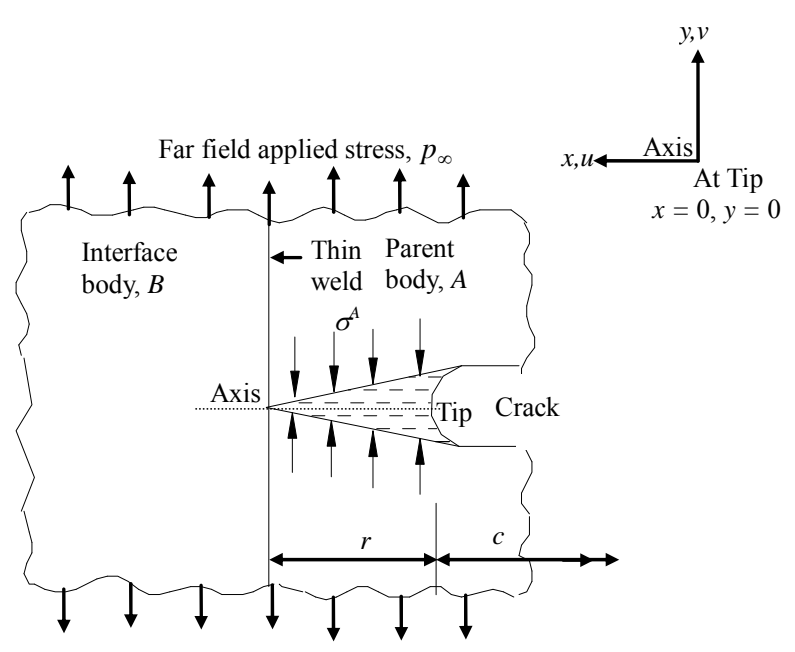

(a)

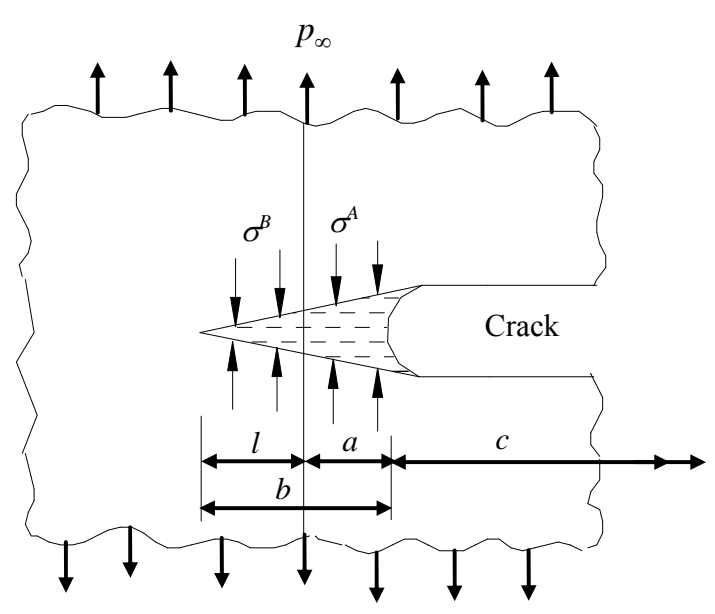

(b)

Figure 2. Stages of crack advancement towards a thin weld; (a) Stage I (Cohesive zone in parent body); (b) Stage II (Extension of cohesive zone into interface body).

$$
v(x)-\frac{2}{E}\left[\int_{0}^{x} \frac{K_{\text {applied }}}{\sqrt{2 \pi(r-x)}} \mathrm{d} x+\int_{0}^{x} \frac{\mathrm{d} x}{\pi \sqrt{r-x}} \int_{0}^{r} \frac{\sigma^{A} \sqrt{r-\xi}}{\xi-x} \mathrm{~d} \xi\right]
$$

Stage I is valid till the cohesive zone lies in the parent body i.e. the distance of the crack tip from the interface, $a$, fulfills the condition, $a \geq r$.

Refer Figure 2(b). The crack has grown ahead from Stage I such that $a<r$. The cohesive zone has developed in interface body, $B$, with its extent up to distance, $l$, from the interface. Length of the cohesive zone, $b$, is equal to $(a+l)$. Since, $E^{A}=E^{B}=E$, the following expression is written for $v(x)$ in Stage II under simultaneous action of different cohesive stresses $\sigma^{A}$ and $\sigma^{B}$ in parent and interface bodies respectively with the help of Equation (2) 


$$
v(x)=-\frac{2}{E}\left[\int_{0}^{x} \frac{K_{\text {applied }}}{\sqrt{2 \pi(b-x)}} \mathrm{d} x+\int_{0}^{x} \frac{\mathrm{d} x}{\pi \sqrt{b-x}} \int_{0}^{a} \frac{\sigma^{A} \sqrt{b-\xi}}{\xi-x} \mathrm{~d} \xi+\int_{0}^{x} \frac{\mathrm{d} x}{\pi \sqrt{b-x}} \int_{a}^{b} \frac{\sigma^{B} \sqrt{b-\xi}}{\xi-x} \mathrm{~d} \xi\right]
$$

\subsection{Crack Near Thick Weld (Refer Figure 3)}

Using similar principles as in thin weld, the expressions

$$
\begin{aligned}
& v(x)=-\frac{2}{E}\left[\int_{0}^{x} \frac{K_{\text {applied }}}{\sqrt{2 \pi(r-x)}} \mathrm{d} x+\int_{0}^{x} \frac{\mathrm{d} x}{\pi \sqrt{r-x}} \int_{0}^{r} \frac{\sigma^{A} \sqrt{r-\xi}}{\xi-x} \mathrm{~d} \xi\right] \\
& v(x)=-\frac{2}{E}\left[\int_{0}^{x} \frac{K_{\text {applied }}}{\sqrt{2 \pi(b-x)}} \mathrm{d} x+\int_{0}^{x} \frac{\mathrm{d} x}{\pi \sqrt{b-x}} \int_{0}^{a} \frac{\sigma^{A} \sqrt{b-\xi}}{\xi-x} \mathrm{~d} \xi+\int_{0}^{x} \frac{\mathrm{d} x}{\pi \sqrt{b-x}} \int_{a}^{b} \frac{\sigma^{B} \sqrt{b-\xi}}{\xi-x} \mathrm{~d} \xi\right] \\
& v(x)=-\frac{2}{E}\left[\int_{0}^{x} \frac{K_{\text {applied }}}{\sqrt{2 \pi(b-x)}} \mathrm{d} x+\int_{0}^{x} \frac{\mathrm{d} x}{\pi \sqrt{b-x}} \int_{0}^{a} \frac{\sigma^{A} \sqrt{b-\xi}}{\xi-x} \mathrm{~d} \xi+\int_{0}^{x} \frac{\mathrm{d} x}{\pi \sqrt{b-x}} \int_{a}^{b_{1}} \frac{\sigma^{W} \sqrt{b-\xi}}{\xi-x} \mathrm{~d} \xi+\int_{0}^{x} \frac{\mathrm{d} x}{\pi \sqrt{b-x}} \int_{b_{1}}^{b} \frac{\sigma^{B} \sqrt{b-\xi}}{\xi-x} \mathrm{~d} \xi\right]
\end{aligned}
$$

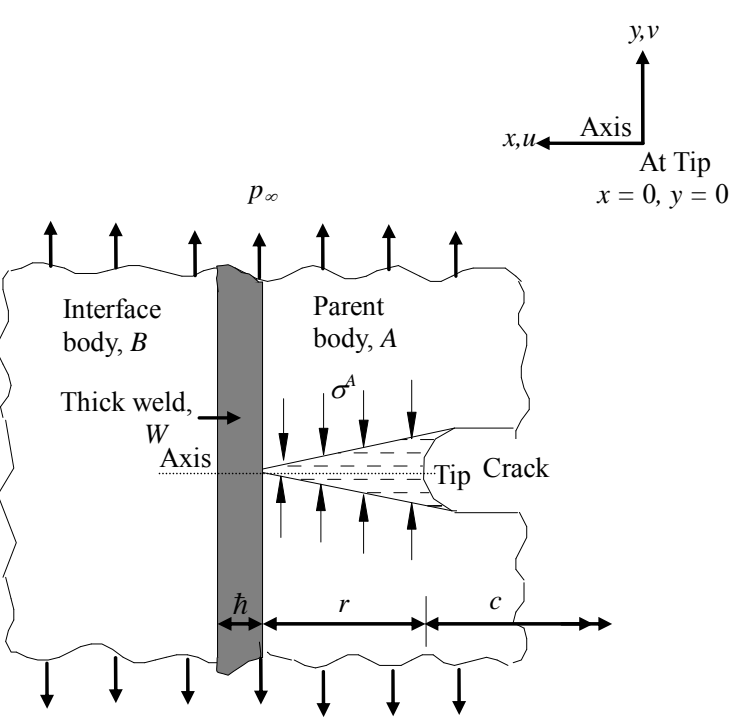

(a)

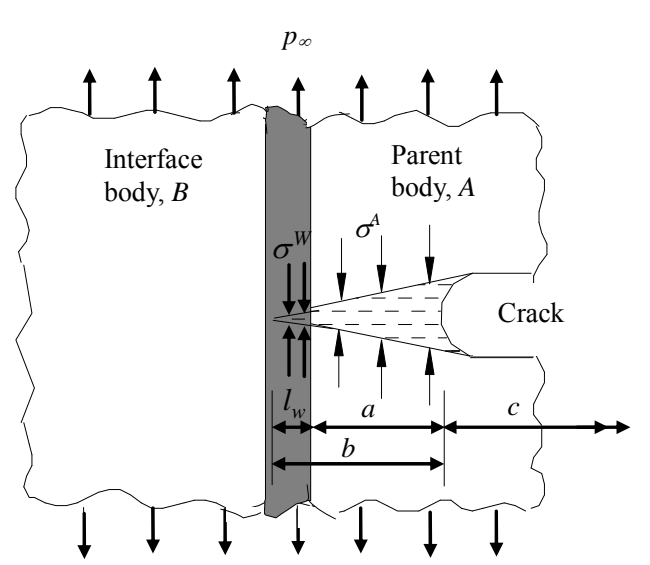

(b)

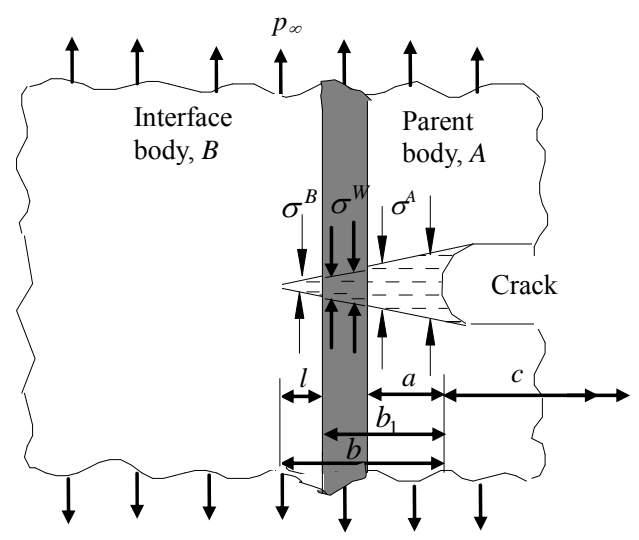

(c)

Figure 3. Stages of crack advancement towards a thick weld; (a) Stage I (Cohesive zone in parent body); (b) Stage II (Spread of cohesive zone to weld); (c) Stage III (Extension of cohesive zone into interface body). 
All the integrals, especially non-singular ones, in Equation (1) to Equation (6) need to be solved for obtaining a usable form of $v(x)$.

\section{Solution}

Equations in important stages of crack growth towards the interface in bodies with thin and thick welds are solved as follows.

\subsection{Crack Near Thin Weld}

In Stage II,

$$
v(x)=\frac{2}{E}\left[I_{1}+I_{2}+I_{3}\right]
$$

where

$$
\begin{aligned}
& I_{1}=-\int_{0}^{x} \frac{K_{\text {applied }}}{\sqrt{2 \pi(b-x)}} \mathrm{d} x, \\
& I_{2}=-\int_{0}^{x} \frac{\mathrm{d} x}{\pi \sqrt{b-x}} \int_{0}^{a} \frac{\sigma^{A} \sqrt{b-\xi}}{\xi-x} \mathrm{~d} \xi,
\end{aligned}
$$

and

$$
I_{3}=-\int_{0}^{x} \frac{\mathrm{d} x}{\pi \sqrt{b-x}} \int_{a}^{b} \frac{\sigma^{B} \sqrt{b-\xi}}{\xi-x} \mathrm{~d} \xi
$$

Integral $I_{1}$ is easily solved as

$$
I_{1}=K_{\text {applied }} \sqrt{\frac{2}{\pi}(b-x)}
$$

Integrals $I_{2}$ and $I_{3}$ are singular in nature and are evaluated as follows:

$$
I_{2}=-\frac{\sigma^{A}}{\pi} \int_{0}^{x} \frac{\mathrm{d} x}{\sqrt{b-x}} \int_{0}^{a} \frac{\sqrt{b-\xi}}{\xi-x} \mathrm{~d} \xi,
$$

Use of Cauchy's principal value theorem [3] enables to write for $0<x<a$ and continuous $\sqrt{b-\xi}$ as

$$
I_{2}=-\frac{\sigma^{A}}{\pi} \int_{0}^{a} \sqrt{b-\xi} \mathrm{d} \xi \int_{0}^{x} \frac{1}{\sqrt{b-x}(\xi-x)} \mathrm{d} x .
$$

On using the following substitutions

$$
\begin{aligned}
& b-x=C_{3}^{2} \Rightarrow-\mathrm{d} x=2 C_{3} d C_{3} \Rightarrow-\frac{\mathrm{d} x}{C_{3}} \\
& =2 \mathrm{~d} C_{3} \Rightarrow-\frac{\mathrm{d} x}{\sqrt{b-x}}=2 \mathrm{~d} C_{3}
\end{aligned}
$$

in integral $I_{2}$, one obtains

$$
I_{2}=-\frac{2 \sigma^{A}}{\pi} \int_{0}^{a} \sqrt{b-\xi} \mathrm{d} \xi \int_{\sqrt{b}}^{\sqrt{b-x}} \frac{1}{\left(b-\xi-C_{3}^{2}\right)} \mathrm{d} C_{3}
$$

On evaluating the inner integral, one obtains

$$
I_{2}=-\frac{2 \sigma^{A}}{\pi} \int_{0}^{a}\left(\tanh ^{-1} \frac{\sqrt{b-x}}{\sqrt{b-\xi}}-\tanh ^{-1} \frac{\sqrt{b}}{\sqrt{b-\xi}}\right) \mathrm{d} \xi
$$

Since for + ve $\xi$, term $\tanh ^{-1} \frac{\sqrt{b}}{\sqrt{b-\xi}}$ causes singularity in the solution and is not considered further. Therefore

$$
I_{2}=-\frac{2 \sigma^{A}}{\pi} \int_{0}^{a}\left(\tanh ^{-1} \frac{\sqrt{b-x}}{\sqrt{b-\xi}}\right) \mathrm{d} \xi
$$

which can also be written as

$$
I_{2}=-\frac{\sigma^{A}}{\pi} \int_{0}^{a}[\ln (\sqrt{b-\xi}+\sqrt{b-x})-\ln (\sqrt{b-\xi}-\sqrt{b-x})] \mathrm{d} \xi
$$

On defining $\sqrt{b-\xi}=C_{4}$, one obtains $\mathrm{d} \xi=-2 C_{4} \mathrm{~d} C_{4}$ Therefore

$$
\int \ln (\sqrt{b-\xi}+\sqrt{b-x}) \mathrm{d} \xi=\int-2 C_{4} \ln \left(C_{4}+C_{5}\right) \mathrm{d} C_{4}
$$

where constant $C_{5}=\sqrt{b-x}$. Solution of the integral $\int-2 C_{4} \ln \left(C_{4}+C_{5}\right) \mathrm{d} C_{4} \quad$ is obtained as

$$
-2 C_{4}\left(C_{4}+C_{5}\right)\left[\ln \left(C_{4}+C_{5}\right)-1\right]+\left(C_{4}+C_{5}\right)^{2}\left[\ln \left(C_{4}+C_{5}\right)-1\right]-\frac{1}{2}\left(C_{4}+C_{5}\right)^{2}
$$

Similarly, other term $\int \ln (\sqrt{b-\xi}-\sqrt{b-x}) \mathrm{d} \xi$ in integral $I_{2}$ is obtained as

$$
-2 C_{4}\left(C_{4}-C_{5}\right)\left[\ln \left(C_{4}-C_{5}\right)-1\right]+\left(C_{4}-C_{5}\right)^{2}\left[\ln \left(C_{4}-C_{5}\right)-1\right]-\frac{1}{2}\left(C_{4}-C_{5}\right)^{2}
$$

Integral $I_{2}$ is finally written as 


$$
\begin{aligned}
I_{2}= & -\frac{\sigma^{A}}{\pi}\left[\left\{-2 C_{4}\left(C_{4}+C_{5}\right)\left[\ln \left(C_{4}+C_{5}\right)-1\right]+\left(C_{4}+C_{5}\right)^{2}\left[\ln \left(C_{4}+C_{5}\right)-1\right]-\frac{1}{2}\left(C_{4}+C_{5}\right)^{2}\right\}\right. \\
& \left.-\left\{-2 C_{4}\left(C_{4}-C_{5}\right)\left[\ln \left(C_{4}-C_{5}\right)-1\right]+\left(C_{4}-C_{5}\right)^{2}\left[\ln \left(C_{4}-C_{5}\right)-1\right]-\frac{1}{2}\left(C_{4}-C_{5}\right)^{2}\right\}\right]_{0}^{a}
\end{aligned}
$$

On re-substituting $C_{4}$ and $C_{5}$, one obtains

$$
I_{2}=-\frac{\sigma^{A}}{\pi}\left[(b-\xi) \ln \left(\frac{\sqrt{b-\xi}-\sqrt{b-x}}{\sqrt{b-\xi}+\sqrt{b-x}}\right)+(b-x) \ln \left(\frac{\sqrt{b-\xi}+\sqrt{b-x}}{\sqrt{b-\xi}-\sqrt{b-x}}\right)-2 \sqrt{b-\xi} \sqrt{b-x}\right]_{0}^{a}
$$

Applying the limits of integration results in the following

$$
\begin{aligned}
I_{2} & =\frac{x \sigma^{A}}{\pi} \ln \left[\frac{\sqrt{b}-\sqrt{b-x}}{\sqrt{b}+\sqrt{b-x}}\right]-\frac{2 \sigma^{A}}{\pi} \sqrt{b} \sqrt{b-x}-\frac{\sigma^{A}}{\pi} a \ln \left[\frac{\sqrt{b-a}+\sqrt{b-x}}{|\sqrt{b-x}-\sqrt{b-a}|}\right] \\
& -\frac{\sigma^{A}}{\pi} x \ln \left[\frac{|\sqrt{b-x}-\sqrt{b-a}|}{\sqrt{b-x}+\sqrt{b-a}}\right]+\frac{2 \sigma^{A}}{\pi} \sqrt{(b-a)(b-x)}
\end{aligned}
$$

Similarly, Integral $I_{3}$ is written as

$$
-\frac{\sigma^{B}}{\pi} \int_{a}^{b}[\ln (\sqrt{b-\xi}+\sqrt{b-x})-\ln (\sqrt{b-\xi}-\sqrt{b-x})] \mathrm{d} \xi
$$

and is evaluated in similar manner as integral $I_{2}$.

$$
I_{3}=-\frac{\sigma^{B}}{\pi}\left[(b-\xi) \ln \left(\frac{\sqrt{b-\xi}-\sqrt{b-x}}{\sqrt{b-\xi}+\sqrt{b-x}}\right)+(b-x) \ln \left(\frac{\sqrt{b-\xi}+\sqrt{b-x}}{\sqrt{b-\xi}-\sqrt{b-x}}\right)-2 \sqrt{b-\xi} \sqrt{b-x}\right]_{a}^{b}
$$

On applying the limits of integration, one obtains

$$
I_{3}=\frac{a \sigma^{B}}{\pi} \ln \left[\frac{\sqrt{b-a}+\sqrt{b-x}}{|\sqrt{b-x}-\sqrt{b-a}|}\right]+\frac{x \sigma^{B}}{\pi} \ln \left[\frac{|\sqrt{b-x}-\sqrt{b-a}|}{\sqrt{b-a}+\sqrt{b-x}}\right]-\frac{2 \sigma^{B}}{\pi} \sqrt{(b-a)(b-x)}
$$

Substitution of integrals $I_{1}, I_{2}$ and $I_{3}$ in Equation (7) on using, $b=a+l$, results in closed form expression for

load line displacement, $v(x)$, in the cohesive zone spread across the interface of bodies $A$ and $B$ as

$$
\begin{aligned}
v(x) & =\frac{2}{E}\left[K_{\text {applied }} \sqrt{\frac{2}{\pi}(a+l-x)}+\frac{\sigma^{A}}{\pi}\left[x \ln \left(\frac{\sqrt{(a+l)}-\sqrt{(a+l-x)})}{\sqrt{(a+l)}+\sqrt{(a+l-x)}}\right)-2 \sqrt{(a+l)(a+l-x)}\right]\right. \\
& \left.+\frac{\sigma^{B}-\sigma^{A}}{\pi}\left[a \ln \left(\frac{\sqrt{(a+l-x)}+\sqrt{l}}{|\sqrt{(a+l-x)}-\sqrt{l}|}\right)+x \ln \left(\frac{|\sqrt{(a+l-x)}-\sqrt{l}|}{\sqrt{(a+l-x)}+\sqrt{l}}\right)-2 \sqrt{l(a+l-x)}\right]\right]
\end{aligned}
$$

\subsection{Crack Near Thick Weld}

Solution in Stage II is obtained upon replacing $\sigma^{B}$ by $\sigma^{W}$ and $l$ by $l_{w}$ in Equation (8).

In Stage III,

$$
v(x)=\frac{2}{E}\left[I_{1}+I_{2}+I_{3}+I_{4}\right]
$$

where $I_{1}$ and $I_{2}$ are similar as in Equation (7).

$I_{3}$ is written as

$$
I_{3}=-\int_{0}^{x} \frac{\mathrm{d} x}{\pi \sqrt{b-x}} \int_{a}^{b_{1}} \frac{\sigma^{W} \sqrt{b-\xi}}{\xi-x} \mathrm{~d} \xi
$$

or 


$$
I_{3}=-\frac{\sigma^{W}}{\pi}\left[(b-\xi) \ln \left(\frac{\sqrt{b-\xi}-\sqrt{b-x}}{\sqrt{b-\xi}+\sqrt{b-x}}\right)+(b-x) \ln \left(\frac{\sqrt{b-\xi}+\sqrt{b-x}}{\sqrt{b-\xi}-\sqrt{b-x}}\right)-2 \sqrt{b-\xi} \sqrt{b-x}\right]_{a}^{b_{1}}
$$

or

$$
\begin{aligned}
I_{3}= & -\frac{\sigma^{W}}{\pi}\left[-b_{1} \ln \left(\frac{\sqrt{b-b_{1}}-\sqrt{b-x}}{\sqrt{b-b_{1}}+\sqrt{b-x}}\right)-x \ln \left(\frac{\sqrt{b-b_{1}}+\sqrt{b-x}}{\sqrt{b-b_{1}}-\sqrt{b-x}}\right)-2 \sqrt{\left(b-b_{1}\right)(b-x)}\right. \\
& \left.+a \ln \left(\frac{\sqrt{b-a}-\sqrt{b-x}}{\sqrt{b-a}+\sqrt{b-x}}\right)+x \ln \left(\frac{\sqrt{b-a}+\sqrt{b-x}}{\sqrt{b-a}-\sqrt{b-x}}\right)+2 \sqrt{(b-a)(b-x)}\right]
\end{aligned}
$$

$I_{4}$ is written as $I_{4}=-\int_{0}^{x} \frac{\mathrm{d} x}{\pi \sqrt{b-x}} \int_{b_{1}}^{b} \frac{\sigma^{B} \sqrt{b-\xi}}{\xi-x} \mathrm{~d} \xi$

or

$$
I_{4}=-\frac{\sigma^{B}}{\pi}\left[(b-\xi) \ln \left(\frac{\sqrt{b-\xi}-\sqrt{b-x}}{\sqrt{b-\xi}+\sqrt{b-x}}\right)+(b-x) \ln \left(\frac{\sqrt{b-\xi}+\sqrt{b-x}}{\sqrt{b-\xi}-\sqrt{b-x}}\right)-2 \sqrt{b-\xi} \sqrt{b-x}\right]_{b_{1}}^{b}
$$

or

$$
I_{4}=-\frac{\sigma^{B}}{\pi}\left[b_{1} \ln \left(\frac{\sqrt{b-b_{1}}-\sqrt{b-x}}{\sqrt{b-b_{1}}+\sqrt{b-x}}\right)+x \ln \left(\frac{\sqrt{b-b_{1}}+\sqrt{b-x}}{\sqrt{b-b_{1}}-\sqrt{b-x}}\right)+2 \sqrt{\left(b-b_{1}\right)(b-x)}\right]
$$

Substitution of integrals $I_{1}$ to $I_{4}$ in Equation (9) results cohesive zone spread across parent body, $A$, weld, $W$ and in closed form expression for displacement, $v(x)$, in the interface body, $B$, as

$$
\begin{aligned}
& v(x)=\frac{2}{E}\left\{K_{\text {applied }} \sqrt{\frac{2(b-x)}{\pi}}+\frac{\sigma^{A}}{\pi}\left[x \ln \left(\frac{\sqrt{b}-\sqrt{b-x}}{\sqrt{b}+\sqrt{b-x}}\right)-2 \sqrt{b(b-x)}\right]\right. \\
& +\frac{\sigma^{W}-\sigma^{A}}{\pi}\left[a \ln \left(\frac{\sqrt{b-x}+\sqrt{b-a}}{|\sqrt{b-x}-\sqrt{b-a}|}\right)+x \ln \left(\frac{|\sqrt{b-x}-\sqrt{b-a}|}{\sqrt{b-a}+\sqrt{b-x}}\right)-2 \sqrt{(b-a)(b-x)}\right] \\
& \left.+\frac{\sigma^{B}-\sigma^{W}}{\pi}\left[b_{1} \ln \left(\frac{\sqrt{b-x}+\sqrt{b-b_{1}}}{\left|\sqrt{b-x}-\sqrt{b-b_{1}}\right|}\right)+x \ln \left(\frac{\left|\sqrt{b-x}-\sqrt{b-b_{1}}\right|}{\sqrt{b-b_{1}}+\sqrt{b-x}}\right)-2 \sqrt{\left(b-b_{1}\right)(b-x)}\right]\right\}
\end{aligned}
$$

\section{Validation}

Solution of integrals is validated by once again reverting to Stages I and II of crack near thin weld. Refer Equation (2). In Stage I, solution of integral

$$
-\int_{0}^{x} \frac{K_{\text {applied }}}{\sqrt{2 \pi(r-x)}} \mathrm{d} x \text { is } K_{\text {applied }} \sqrt{\frac{2}{\pi}(r-x)} .
$$$$
-\frac{\sigma^{A}}{\pi}\left[(r-\xi) \ln \left(\frac{\sqrt{r-\xi}-\sqrt{r-x}}{\sqrt{r-\xi}+\sqrt{r-x}}\right)+(r-x) \ln \left(\frac{\sqrt{r-\xi}+\sqrt{r-x}}{\sqrt{r-\xi}-\sqrt{r-x}}\right)-2 \sqrt{r-\xi} \sqrt{r-x}\right]_{0}^{r}
$$

which simplifies to

$$
\frac{\sigma^{A}}{\pi}\left[r \ln \left(\frac{\sqrt{r}-\sqrt{r-x}}{\sqrt{r}+\sqrt{r-x}}\right)+(r-x) \ln \left(\frac{\sqrt{r}+\sqrt{r-x}}{\sqrt{r}-\sqrt{r-x}}\right)-2 \sqrt{r} \sqrt{r-x}\right]
$$


Crack tip opening displacement (CTOD), $v(x)$ at $x=0$, i.e., $v(0)$ is obtained as $\frac{2}{E}\left\{K_{\text {applied }} \sqrt{\frac{2 r}{\pi}}-\frac{2 \sigma^{A} r}{\pi}\right\}$. On applying Dugdale's cohesive zone criterion,

$$
r=\frac{\pi}{8}\left(\frac{K_{\text {applied }}}{\sigma^{A}}\right)^{2}, v(0) \text { reduces to } \frac{\left(K_{\text {applied }}\right)^{2}}{2 E \sigma^{A}}
$$

which is the well known solution for half crack tip opening displacement in homogenous body $A$ in linear elastic regime.

In Stage II, $J$ integral at crack tip, $J_{\text {tip }}$, expressed as
$\frac{\left(K_{\text {tip }}\right)^{2}}{E}$, is equal to $2 \sigma^{A} v(0)$ whereas $J$ integral at interface, $J_{\text {interface }}$, is equal to $2\left(\sigma^{B}-\sigma^{A}\right) v(a)$. Since $J_{\text {applied }}$ is given by $\frac{\left(K_{\text {applied }}\right)^{2}}{E}$, the following equation is obtained upon using conservation of energy release rate, $J_{\text {applied }}=J_{\text {tip }}+J_{\text {interface }}$

$$
\frac{\left(K_{\text {applied }}\right)^{2}}{E}=2 \sigma^{A} v(0)+2\left(\sigma^{B}-\sigma^{A}\right) v(a)
$$

Equation (8) leads to the following expressions

$$
v(0)=\frac{2}{E}\left\{K_{\text {applied }} \sqrt{\frac{2}{\pi}(a+l)}-\frac{\sigma^{A}}{\pi}[2(a+l)]+\frac{\sigma^{B}-\sigma^{A}}{\pi}\left[a \ln \left(\frac{\sqrt{(a+l)}+\sqrt{l}}{\sqrt{(a+l)}-\sqrt{l}}\right)-2 \sqrt{l(a+l)}\right]\right\}
$$

and

$$
v(a)=\frac{2}{E}\left\{K_{\text {applied }} \sqrt{\frac{2 l}{\pi}}+\frac{\sigma^{A}}{\pi}\left[a \ln \left(\frac{\sqrt{(a+l)}-\sqrt{l}}{\sqrt{(a+l)}+\sqrt{l}}\right)-2 \sqrt{(a+l)(l)}\right]-2 l\left(\frac{\sigma^{B}-\sigma^{A}}{\pi}\right)\right\}
$$

Stress intensity parameter applied over the crack with its cohesive zone split across the interface in Stage II [4]

$$
K_{\text {applied }}=2 \sigma^{A} \sqrt{\frac{2(a+l)}{\pi}}+2\left(\sigma^{B}-\sigma^{A}\right) \sqrt{\frac{2 l}{\pi}}
$$

With Equation (12) and Equation (13), R.H.S. of Equation (11) results in the expression

$$
\frac{1}{E}\left[\frac{8\left(\sigma^{A}\right)^{2}(a+l)}{\pi}+8\left(\sigma^{B}-\sigma^{A}\right)^{2}\left(\frac{l}{\pi}\right)+\frac{16 \sigma^{A}\left(\sigma^{B}-\sigma^{A}\right)}{\pi} \sqrt{l(a+l)}\right]
$$

which equals $\frac{\left(K_{\text {applied }}\right)^{2}}{E}$ i.e. L.H.S upon using Equation (14) that validates the solutions of singular integrals.

\section{Conclusion}

Singular integrals in mathematical model of load line opening of Mode I crack near the interface of elasticity identical but strength and plasticity mismatched materials in linear elastic regime are solved. The solution is well validated.

\section{Acknowledgements}

Support received from the School of Mechanical Building Sciences, VIT University, Vellore, India during the course of this work is gratefully acknowledged.

\section{REFERENCES}

[1] G. T. Hahn and A. R. Rosenfield, "Local Yielding and Extension of a Crack under Plane Stress," Acta Metallurgica, Vol. 13, No. 3, 1965, pp. 293-306. doi:10.1016/0001-6160(65)90206-3

[2] D. Wappling, J. Gunnars and P. Stahle, "Crack Growth across a Strength Mismatched Bimaterial Interface," International Journal of Fracture, Vol. 89, No. 3, 1998, pp. 223-243. doi:0.1023/A:1007493028039

[3] D. Porter and D. S. G. Stirling, "Integral Equations-A Practical Treatment, from Spectral Theory to Applications," Cambridge University Press, Cambridge, 1990.

[4] F. O. Reimelmoser and R. Pippan, "The J-Integral at Dugdale Cracks Perpendicular to Interfaces of Materials with Dissimilar Yield Stresses," International Journal of Fracture, Vol. 103, No. 4, 2000, pp. 397-418. doi:10.1023/A:1007605224764 